\title{
El papel de las cooperativas de crédito en el sostenimiento de la España vaciada a través del capital social
}

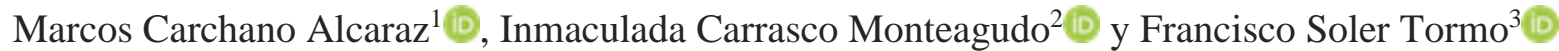

Recibido: 15 de septiembre de 2020 / Aceptado: 2 de diciembre de 2020 / Publicado: 10 de marzo de 2021

Resumen. El sistema bancario viene experimentando grandes transformaciones, marcadas por la disminución del número de entidades y la concentración del negocio. Las cooperativas de crédito han logrado mantenerse relativamente aisladas de las turbulencias financieras de la pasada crisis gracias a políticas de inversión prudentes y la clara vocación territorial y de proximidad con el entorno, características que, junto con su carácter cooperativo, se han convertido en las principales fortalezas de este tipo de entidades de crédito.

Por otra parte, la creciente desatención del sistema bancario hacia los territorios en retroceso demográfico ha propiciado la exclusión financiera de sus habitantes. En este contexto, el objetivo de este trabajo consiste en presentar la importancia de las cooperativas de crédito en España, sobre todo en aquellas provincias de menor densidad de población. Para ello, se presenta un estudio que pone de manifiesto la estrecha relación existente entre las cooperativas de crédito y la financiación del desarrollo regional. Las cooperativas de crédito, a costa de su menor rentabilidad económica, se ponen al servicio de las necesidades de su entorno, vinculándose con el mismo e integrándose en su entramado asociativo, lo que acentúa el papel fundamental que juegan en la lucha contra la exclusión financiera por medio de la formación de capital social.

Palabras clave: Cooperativas de crédito; Economía social; Exclusión financiera; Despoblación; Desarrollo regional. Claves Econlit: P13; R58; B55; A13; R23.

\section{[en] The role of credit cooperatives supporting empty Spain through social capital}

\begin{abstract}
The banking sector has been suffering huge transformations due to fall down of the number of credit institutions and the business' concentration. The credit cooperatives have achieved to keep them self rather isolated of the financial turmoil thanks to the prudence investment polies and their territorial and proximity vocation with the environment. These traits, together with the cooperative character, are the main strengths of credit cooperatives.

On the other hand, the growing inattention of banking sector towards territories in demographic decline, has led to the financial exclusion of their neighbors. In this context, the objective of this work is to present the credit cooperatives' importance in Spain, deepen in provinces with lower population density. To achieve this goal, we have done a study that reveals the strong relationship between credit cooperatives and regional development. At the expense of their lowest profitability, credit cooperatives come forward to serve the needs of their environment, linking with it and integrating into its associative network, which accentuates their role in the fight against financial exclusion by means of social capital formation.
\end{abstract}

Keywords: Credit cooperatives; Social economy; Financial exclusion; Depopulation; Regional development.

Sumario. 1. Introducción. 2. Capital social: concepto y relación con la cooperación. 3. Características socioeconómicas de la España vaciada. 4. El papel de las cooperativas de crédito en la España vaciada. 5. Conclusiones. 6. Referencias bibliográficas.

Cómo citar. Carchano Alcaraz, M.; Carrasco Monteagudo, I.; Soler Tormo, F. (2021) El papel de las cooperativas de crédito en el sostenimiento de la España vaciada a través del capital social. REVESCO. Revista de Estudios Cooperativos, vol. 138 , e73866. https://dx.doi.org/10.5209/reve.73866.

1 Universidad de Castilla-La Mancha, España.

Dirección de correo electrónico: marcos.carchano@alu.uclm.es.

2 Universidad de Castilla-La Mancha, España.

Dirección de correo electrónico: inmaculada.carrasco@uclm.es.

3 Universidad de Valencia, España.

Dirección de correo electrónico: francisco.soler@uv.es. 


\section{Introducción}

La reciente crisis financiera ha impactado intensamente al sistema bancario, provocando un fuerte retroceso en la actividad crediticia, lo que ha dificultado la recuperación económica. Los escándalos financieros y la aparente impunidad frente a comportamientos predadores, ha espoleado la demanda de formas diferentes de hacer banca y ha hecho volver la mirada hacia entidades pequeñas, de vocación territorial, que han resistido con mayor firmeza al colapso crediticio: las cooperativas. Asimismo, surge una renovada atención hacia el sector primario $\mathrm{y}$, paralelamente, hacia la desatendida "España vaciada" cuyo papel parece haberse revalorizado y percibido como realmente estratégico.

En este contexto, las cooperativas de crédito, que se han mantenido aisladas de las turbulencias financieras vividas en los años de crisis, vienen adquiriendo un mayor protagonismo en la economía española, representando el $7,4 \%$ del crédito total del sistema bancario y habiendo experimentado una evolución positiva de más de dos puntos porcentuales entre 2008 y 2018. Han sido sus propias características (reducido tamaño, localismo y vocación de banca de proximidad), las razones principales de esta relativa inmunidad ante los efectos nocivos de la crisis financiera. Fruto de esto, se plantea la revalorización del localismo como elemento clave a la hora de otorgar una mayor estabilidad al sistema financiero y contribuir al equilibrio regional (Arnone, 2015; Ammirato, 2018). De hecho, Giagnocavo, Gérez y Sforzi (2012), manifiestan la importancia de las cooperativas de crédito, destacando el caso de España e Italia, donde el asentamiento de éstas ha contribuido a la dinamización regional.

En un intento de evaluar la relevancia del cooperativismo de crédito en el desarrollo de los territorios en retroceso demográfico y resaltar la importancia de su especificidad, tanto por lo que se refiere a su vocación de banca al servicio de un territorio como al respeto a unos valores y principios que distinguen su actividad del negocio de la banca lucrativa, se plantea el presente trabajo, cuya hipótesis de partida es que las cooperativas de crédito han contribuido a frenar la restricción crediticia y en consecuencia los procesos de pérdida de población en aquellas zonas caracterizadas como la "España Vaciada". Con este objetivo, en primer lugar, se plantea un indicador que muestra la relación existente entre la densidad de población y la estructura demográfica, para posteriormente realizar un análisis gráfico de la relación existente entre esta ratio y la cuota de mercado de las cooperativas. Seguidamente, con el mismo objetivo, atendiendo a García Verdugo y De la Cuesta (1999), se plantea un índice de intensidad de crédito y a partir de éste, se calcularán y se compararán las tasas de variación de la intensidad de crédito y la cuota de mercado de las cooperativas para los años 2008-2018, observando que las cooperativas de crédito contribuyen a la lucha contra la exclusión financiera en los territorios más despoblados.

\section{Capital social: concepto y relación con la cooperación}

El desarrollo de una economía depende de su capacidad de producción y ésta, a su vez, de la calidad y disposición de los distintos factores productivos, así como de su eficiencia conjunta. Estos factores incluyen los recursos naturales, el stock de capital físico en sentido amplio y el capital humano, incluyendo la noción de capital intelectual. Ahora bien, la literatura económica ha incorporado un factor adicional denominado "capital social", cuya presencia contribuye al desarrollo económico y social de las regiones (Fukuyama, 1995) y favorece el desarrollo sostenible (Hauberer, 2011), de modo que se pone de manifiesto una relación directa entre la existencia de capital social y los niveles de desarrollo y bienestar de la zona en cuestión (Ferragina, 2012).

Este capital social lo proporciona la habilidad de las personas para trabajar conjuntamente en grupos y organizaciones con el fin de satisfacer propósitos comunes, fruto de su capacidad de cooperar y coordinar esfuerzos (Solow, 2000). En este sentido, el capital social es un intangible que incluye tanto los valores y hábitos compartidos por la población, más o menos heredados e institucionalizados, como el conjunto de redes de organizaciones cívicas, políticas, económicas, asistenciales o religiosas en las que participan los individuos y que permiten identificarse como grupo. Constituye, por tanto, un aglutinante que proporciona cohesión al colectivo y le permite afrontar desafíos conjuntos.

El concepto nació en el ámbito de la sociología y fue inicialmente aplicado al estudio de la decadencia de las comunidades indígenas americanas, en las que se constató la necesidad de conservar valores, objetivos e instituciones compartidos (Hanifan, 1916, citado en Putnam, 2003). Posteriormente se amplió para evaluar la calidad de la democracia en una sociedad (Almond y Verba, 1963; Putnam, 1993), la consolidación del capital humano y de la conciencia cívica (Coleman, 1988), y al ámbito del desarrollo económico y al mundo del microcrédito, a partir de Fukuyama (1995).

El capital social tiene una naturaleza multidimensional, lo que dificulta la existencia de una definición unánime. Los antecedentes del concepto de capital social, tal y como lo conocemos actualmente, los encontramos en investigaciones centradas en estudiar las relaciones entre asociaciones, corporaciones e individuos datadas en 1835 (Inkpen y Tsang, 2005). Así, la primera aproximación a este concepto la 
encontramos en el trabajo de Bourdieu (1980), que define el capital social como "el total de los recursos reales o potenciales que están vinculados a la posesión de una red duradera de relaciones más o menos institucionalizadas de conocimiento mutuo o reconocimiento". Así, teniendo en cuenta esta primera aproximación, son muchos los autores que destacan la importancia de la confianza, la reciprocidad y la asistencia mutua (Fukuyama, 1995; Putman, 1993), pues el capital social, se relaciona con el establecimiento de redes sociales que crean valor fruto de la cooperación entre individuos basada en la existencia de valores como la honestidad, la reciprocidad y la confianza (Carrasco, 2005). Por su parte, Coleman (1990) entiende el capital social como el conjunto de recursos sociales inherentes a la organización social que promueven la acción coordinada. Se puede etiquetar en tres niveles (Sabatini, 2009): los vínculos de parentesco o familiares (bonding social capital), las redes de amistad o de conocimiento (bridging social capital) y los vínculos de sociabilidad y de asociacionismo, tanto de carácter económico, político o cultural (linking social capital). La existencia de un entramado asociativo permite reforzar las relaciones sociales y económicas de los miembros de una comunidad, favorecer su participación y generar una cohesión y una conciencia territoriales, en especial en áreas rurales (Pisani et al., 2017; Carrasco y Buendía, 2013 y 2020; Bretos et al, 2018).

Teniendo en cuenta lo anterior, el capital social se considera, así, un factor que puede contribuir a la capacidad de generar beneficios futuros, por su relevancia en la construcción de la confianza y, por tanto, al éxito de los negocios y a la eficiencia productiva. La existencia de capital social permite la creación de ventajas competitivas, mejora el funcionamiento y resultados de las empresas ( $\mathrm{Wu}, 2008$ ), y favorece en última instancia el bienestar económico, dado el efecto positivo que pueden llegar a tener sobre la comunidad en su conjunto (Sabatini et al., 2014). De este modo, el capital social permite mejorar los resultados en los procesos de desarrollo socioeconómico, favoreciendo la eficiencia del capital humano y financiero, entre otros (Foronda-Robles y Galindo-Pérez-de-Azpillaga, 2012). En este sentido, se establece una relación causal directa entre capital social y desarrollo económico (Devesa, 2011). En esta misma línea, un adecuado capital social junto con un conveniente marco institucional formal interviene como elemento clave en el fomento de crecimiento económico.

Además, este tipo de capital no solo afecta a las personas inmersas en las organizaciones (redes sociales), sino que es capaz de extender sus beneficios a toda la sociedad a través de la generación de externalidades positivas, poniendo de manifiesto la esencialidad que tiene el capital social en el buen funcionamiento de los sistemas económicos (Castaño, 2005). Tal es su importancia en los procesos de crecimiento económico, que autores como Whiteley (2000) sentencian que el impacto de este tipo de capital sobre éstos es similar al del capital humano, pues el capital social actúa como elemento principal en las espirales de dinámica de desarrollo en las regiones (Sweeney, 2001; Saz-Gil y Gómez-Quintero, 2015).

En este sentido, las cooperativas, en general, constituyen un tipo de instituciones que responden a la noción de capital social por distintas razones (Bauer, Guzmán y Santos, 2012; Jones y Kalmi, 2009; Valentinov, 2004):

- $\quad$ En su origen fueron creadas como respuesta a una necesidad común de un colectivo que recurrió a la autoayuda como mecanismo de satisfacerla mutuamente.

- $\quad$ Responden a unos valores y hábitos compartidos, entre los que destacan la participación y la toma de decisiones de forma democrática.

Priman las personas frente al capital, de forma que la distribución de excedentes, en caso de existir, se efectúa en función de la actividad realizada.

Reservan una parte de sus excedentes para la educación y la promoción cooperativa, difundiendo sus valores como empresa y la cultura de su territorio, asumiendo un compromiso con su sociedad. Este destino es cualitativamente relevante, por cuanto esta identificación refuerza el elemento emocional del capital social y la identificación con su entorno (Chaves y Soler, 2005).

Originalmente se circunscribían a entornos limitados, normalmente locales, que fueron ampliándose por la vía de la interoperación entre entidades semejantes. Aun así, suelen mantener una estrecha identificación con su territorio.

Tienden a consolidar unas redes perdurables que incluyen distintos ámbitos que engloban el mercado social: cooperativas de producción, de comercialización, de consumo, de servicios y de crédito.

Frecuentemente, la cooperativa agraria es la empresa más relevante e influyente en muchos de los pequeños y medianos municipios rurales españoles. Muchas de ellas crearon en su seno secciones de crédito para las necesidades financieras de sus socios, en especial en las comunidades autónomas de Valencia, Cataluña y Andalucía (Sanchis y Melián, 2011). Más frecuentemente, impulsaron la creación de cajas rurales conjuntamente a nivel local, comarcal, provincial o superior, reforzando los vínculos que las aglutinan y generando confianza social (Sabatini, Modena y Tortia, 2013). De hecho, el cuestionario del Banco Mundial para la evaluación del capital social incluye tanto las cooperativas agrarias como las de ahorro y crédito (Grootaert et al., 2004). 
En el caso español, y más desde la desaparición de la gran mayoría de las cajas de ahorros, las cooperativas de crédito han reforzado su papel como instituciones arraigadas en el entramado asociativo regional. Con ello contribuyen a robustecer las relaciones de confianza dentro del territorio, como banca de proximidad comprometida con su entorno y su clientela (Seguí-Mas y Server, 2010, Sanchis y Campos, 2007). Estos vínculos facilitan la financiación del desarrollo económico de la zona de actuación (Server y Melián, 1998; Rodríguez, 2006) y, además, constituyen una de las ventajas competitivas de este tipo de entidades de crédito, para las que el capital social supone un elemento fundamental en la generación de beneficios futuros (Seguí-Mas y Server, 2010). En esta misma línea, los autores anteriores señalan que, las cooperativas de crédito cuentan con un conjunto de activos intangibles que participan en la red de relaciones sociales que se dan dentro de su actividad.

De este modo, teniendo en cuenta la definición de cooperativas aportada por Valentinov (2004), el capital social pasa a ser uno de los pilares fundamentales dentro de las cooperativas de crédito, pues éstas cuentan con la capacidad de generar capital social de unión, de aproximación y de vinculación (Bretos et al., 2018), fruto de sus valores, basados en la reciprocidad, confianza (Hogeland, 2006) y la elevada interdependencia de los miembros ante la propiedad conjunta de la empresa (Putman, 2000), entre otros. Así, teniendo en cuenta las conclusiones a las que llegan Seguí-Mas y Server (2010), las cooperativas de crédito deberán centrarse en fortalecer las ventajas competitivas relacionadas con su capital social para su supervivencia dada la hipercompetitividad existente en el sector bancario. Por ello, su actuación no se limita a la consecución de resultados económicos, sino que dan valor también a la satisfacción de las necesidades financieras de sus socios (Palomo y Sanchis, 2008; Sanchis y Melián, 2009).

Las cooperativas deben ser entendidas como sociedades plurales, donde la participación de sus socios y la vigencia de unos valores éticos compartidos como la transparencia, la comunicación o la involucración en la toma de decisiones, son sus principales fortalezas. Esto les facilita establecer relaciones sociales sólidas y estables (Bretos y Errasti, 2017; Bretos et al., 2018), que a su vez se ven reforzadas por los vínculos que mantienen con el resto de las empresas del territorio (Bauer et al., 2012). En este sentido, el cooperativismo de crédito ha contribuido a reforzar las relaciones entre el asociacionismo dentro de su ámbito de actuación, en especial el agrario, desarrollando en muchas ocasiones alianzas entre cajas rurales y cooperativas agrarias (Melián, Campos y Sanchis, 2011). De hecho, las cerca de 3.700 cooperativas agroalimentarias españolas, que aglutinan a cerca de 1.200.000 socios, conforman el núcleo básico de los órganos de gobierno de la práctica totalidad de las cajas rurales.

Hasta ahora hemos destacado la importancia que tienen las cooperativas en la generación de capital social a nivel local. Sin embargo, siguiendo a Carrasco y Buendía (2013), observamos que el capital social determina el establecimiento de cooperativas, de forma que, a mayor nivel de capital social, mayor presencia cooperativa. Este hecho, unido a lo expuesto en los párrafos anteriores, pone de manifiesto la interdependencia existente entre capital social y cooperativas, sobre todo en el ámbito local. En esta línea, centrándonos en la presencia del cooperativismo de crédito, podemos llegar a afirmar como hipótesis de partida que en las provincias de la denominada "España vaciada" la existencia de entidades crediticias del tercer sector, con clara vocación territorial, ha contribuido a generar capital social y a mitigar el retroceso del crédito que se ha puesto de manifiesto con la Gran Recesión, pues en España, las cooperativas de crédito han permanecido relativamente al margen de las mayores turbulencias de la crisis, en parte aisladas a causa de sus propias características. Precisamente, estas peculiaridades fueron entendidas durante el período de expansión del crédito como unas "peligrosas debilidades" que dificultaban participar de pleno en la exuberancia financiera.

\section{Características socioeconómicas de la España vaciada}

La despoblación, entendida como fenómeno demográfico y territorial, es uno de los principales problemas a los que se enfrenta la mayor parte de la España rural, como pone de manifiesto el Instituto Nacional de Estadística, al constatar que el 63\% de los municipios han perdido población entre 2000 y 2018.

Este fenómeno, que se lleva gestando en nuestro país desde hace décadas, tiende a generar una división del territorio en dos velocidades. De un lado, las áreas urbanas y periurbanas que ganan población fruto de su mayor dinamismo económico. Por otro lado, los territorios situados en el centro de la península, mesetas y zonas montañosas, que cuentan con bajas densidades de población, y que constituyen la denominada "España rural". En estos últimos espacios, se produce un círculo vicioso entre reducido crecimiento económico y despoblación, de modo que el retroceso demográfico será más intenso en las zonas rurales con escaso dinamismo económico. Extremadura, las dos Castillas, Aragón y Andalucía serán las áreas más afectadas por este fenómeno si tenemos en cuenta los datos ofrecidos por Consejo Económico y Social (CES, 2018).

Sin embargo, a pesar de que los datos agregados muestran indicios de recuperación demográfica en el medio rural, gran parte de este territorio sigue presentando un declive demográfico profundo, que será más 
intenso en los próximos años si tenemos en cuenta los recientes datos sobre la proyección de población del INE (2018). Concretamente, las provincias de Palencia, Guadalajara y Segovia cuentan en la actualidad con una tasa de riesgo de extinción demográfica cercana al $90 \%$, mientras que en Cuenca y Huesca esta misma tasa se sitúa en torno al 87\% (Miner, 2018).

En este contexto, existen distintos indicadores para delimitar esta dicotomía. En nuestro caso utilizaremos un indicador que conjuga en cierta medida la densidad de población y la estructura demográfica-laboral, como es la ratio de empleos por kilómetro cuadrado. En consecuencia, a efectos de este estudio, se incluyen en la "España vaciada" aquellas provincias en las que existen menos de 20 empleos por kilómetro cuadrado. Así, atendiendo a la imagen 1, quedarían dentro de nuestro estudio las provincias de Albacete, Ávila, Badajoz, Burgos, Cáceres, Ciudad Real, Cuenca, Guadalajara, Jaén, Huesca, León, Lleida, Lugo, Ourense, Palencia, Salamanca, Segovia, Soria, Teruel, Toledo y Zamora; lo que englobaría a 21 de las 50 provincias (dejando al margen las ciudades de Ceuta y Melilla), que suponen el 55,47\% de la superficie total y el $15,10 \%$ de la población de 2018.

Como rasgo común a todas las provincias que se enmarcan en la "España Vaciada" destacamos la baja densidad de población $\left(25,2 \mathrm{hab} / \mathrm{km}^{2}\right)$, en comparación con la media española, situada en $93 \mathrm{hab} / \mathrm{km}^{2}$, que junto con el resto de los problemas sociodemográficos que padecen como el envejecimiento de la población, aislamiento geográfico, ausencia de infraestructuras (servicios sociales, transporte o internet), entre otros, causan una progresiva disminución del peso de estas provincias sobre la población total, habiendo descendido dos puntos porcentuales en los últimos veinte años. Esta situación se hace extensiva al ámbito económico, donde el peso de la "España Vaciada" en el Producto Interior Bruto total es cada vez menor, situándose en 12,9\% para el año 2018, lo que pone de manifiesto el declive económico y demográfico que vienen experimentando estas provincias.

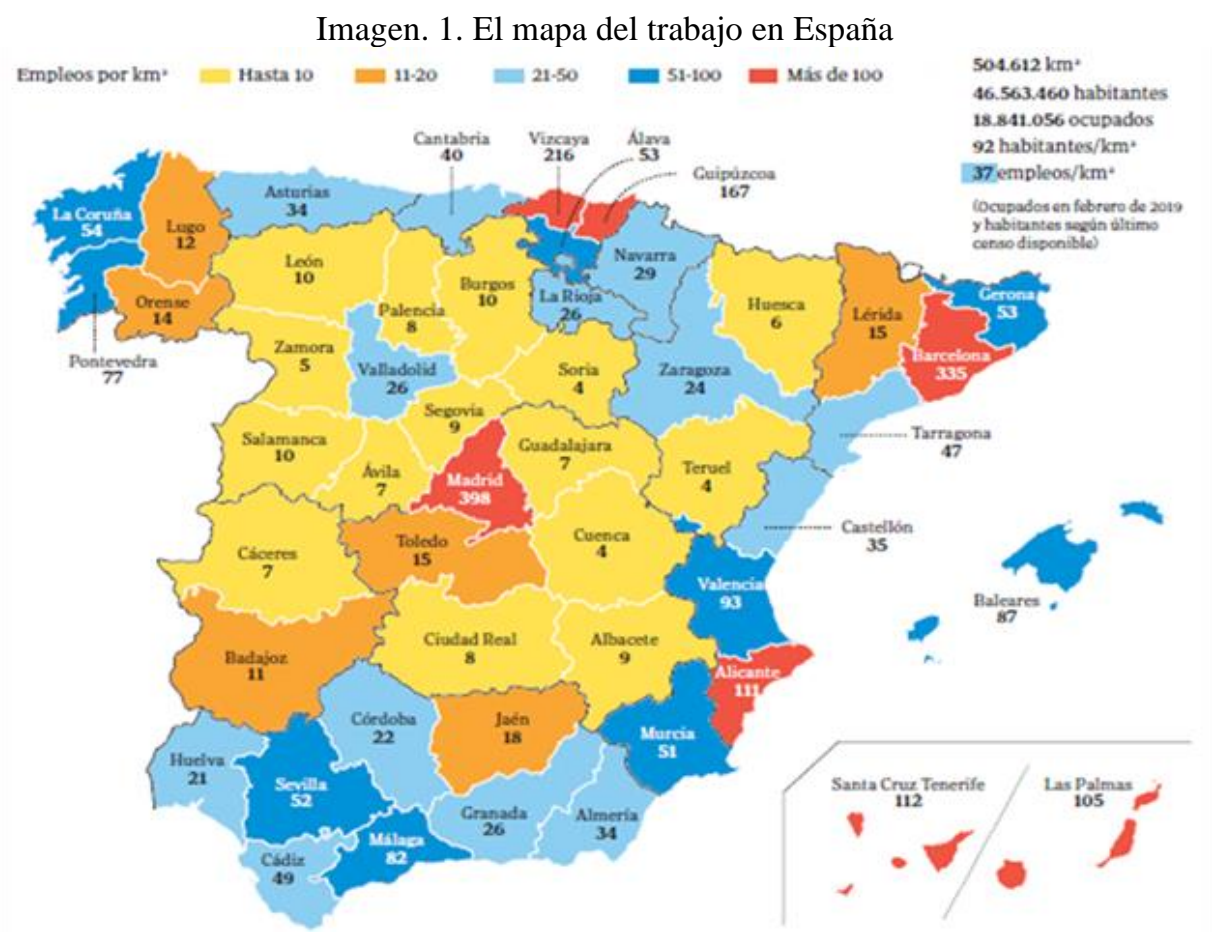

Fuente: ABC, 11/03/2019. Elaborado con datos del INE y de la Seguridad Social

La situación descrita con anterioridad se hace extensiva a la mayor parte de los países europeos (vid Imagen 2) y otros más alejados como Polonia, Rusia, Japón, Estados Unidos, Italia, Alemania o Grecia (Johnson et al, 2015; García y Espejo, 2019; Camarero y Sampedro, 2019; Surchev, 2010; Rajovic y Bulatovic, 2013; Haub y Toshiko, 2014). 
Imagen. 2. Despoblación en Europa $\left(\mathrm{Hab} / \mathrm{Km}^{2}\right)$

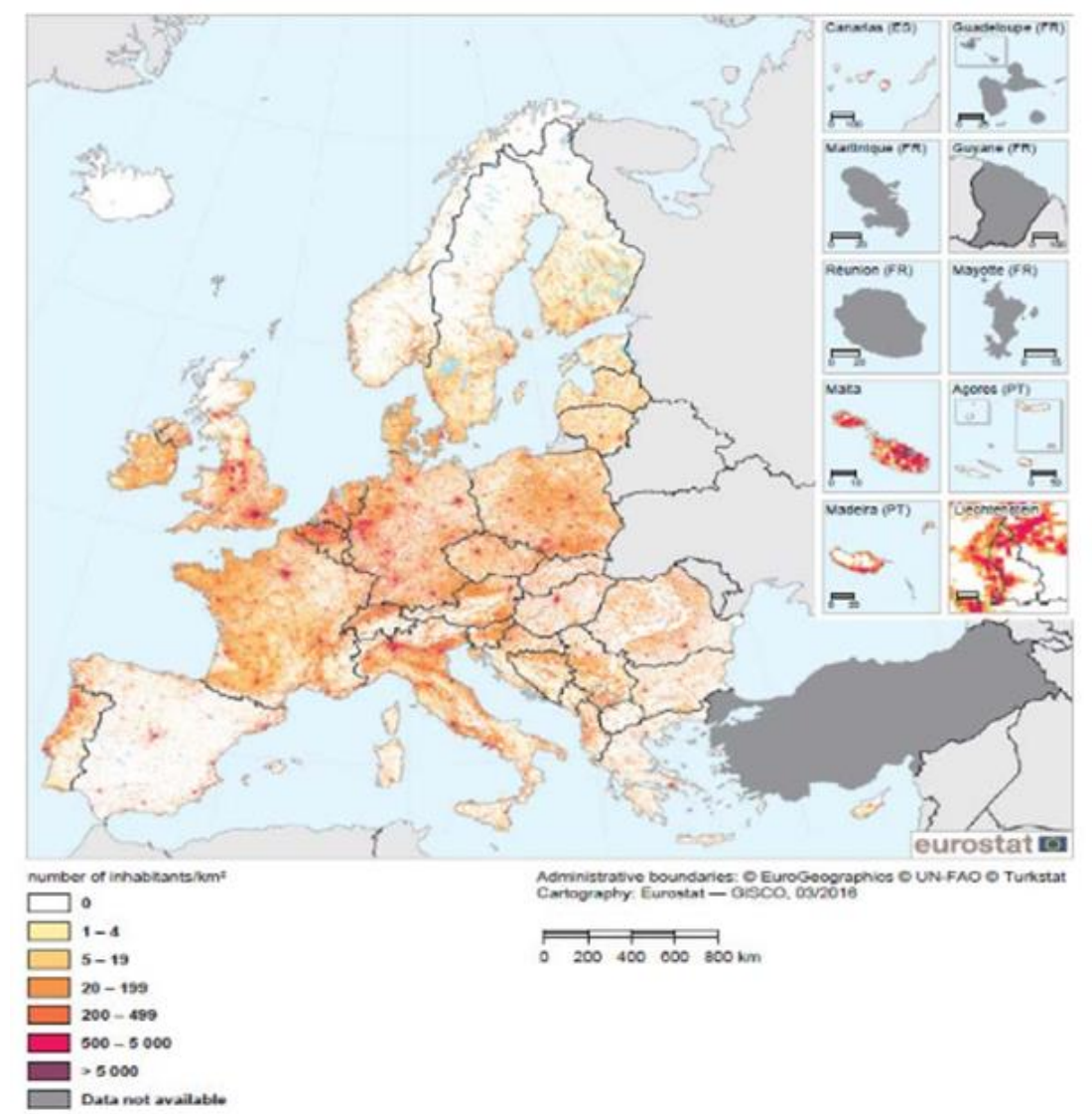

Fuente: Vasilis Margaras. Demographic trends in EU regions. EPRS | European Parliamentary Research Service (2019)

En los últimos años, la pérdida de población responde, por un lado, al bajo crecimiento vegetativo, que se ha convertido en la principal causa de despoblación de las zonas rurales (Ayuda et al, 2000; Garrido y Faci, 2004; Fernández y Meixide, 2010), motivado por la reducción de las tasas de natalidad e intensificado por el creciente envejecimiento de la población (Pérez, 2006; Gómez-Limón et al., 2007); y por otro, a la migración experimentada hacia núcleos de población más dinámicos. Estos movimientos migratorios son más acusados en los colectivos de jóvenes y de mujeres, lo que acentúa al envejecimiento de la población (Pérez y Leco, 2011). Por su parte este fenómeno se puede ver agravado ante la caída de la inmigración extranjera, considerada por algunos autores como contrapunto a la despoblación (Pinilla y Sáez, 2017; Bayona y Gil, 2010; Kasimis, 2010). La situación descrita con anterioridad genera círculos viciosos de crecimiento en las zonas más castigadas por la pérdida de población, como se aprecia en la dotación de centros educativos, considerados por Marré y Rupasingha (2019) como factor de atracción de nuevos residentes. En este sentido, si no existe población suficiente para el establecimiento de este tipo de centros, fruto de la despoblación experimentada en décadas anteriores, no existirán nuevos flujos migratorios que permitan compensar pérdidas de población anteriores, mermando las posibilidades de crecimiento de estas zonas y acentuando la decadencia demográfica.

En este sentido, las cooperativas de crédito como entidades ligadas a la Economía Social se posicionan como un elemento esencial en el freno de la despoblación (Carchano y Carrasco, 2020), ya que actúan como agentes dinamizadores de los recursos y activos locales (Eversole et al., 2013), favoreciendo el desarrollo de las zonas rurales (Mozas y Bernal, 2006), a través de la creación de puestos de trabajo estables y de calidad (Calderón y Calderón, 2012), el apoyo a otras empresas basadas principalmente en la agricultura, distribución comercial, vivienda o trabajo asociado (Chaves y Monzón, 2007) y la generación de actividad económica en general (Tomás et al, 1998). De esta forma, las cooperativas de crédito contribuyen a la dinamización económica de las regiones (Giagnocavo et al., 2012), al mismo tiempo que contribuyen al bienestar social (Azmi, 2015). Igualmente, Ureña et al. (2013), destacan el papel clave que tienen las cooperativas de crédito en el logro de la completa inclusión financiera. 


\section{El papel de las cooperativas de crédito en la España vaciada}

En España, al cierre del ejercicio 2018, existían 62 cooperativas de crédito. Este tipo de entidades financieras, aparecen vinculadas a actividades productivas concretas, siguiendo una estrategia tradicional de prudencia y proximidad. Prueba de ello es el número de oficinas por tramos de población, pues más del $60 \%$ de las oficinas aparecen en municipios de menos de 25.000 habitantes, de las cuales casi el $40 \%$ se establecen en núcleos de menos de 5.000 habitantes.

En esta misma línea, a principios de 2018 las cooperativas de crédito representaban el 7,4\% del crédito total del sistema bancario, presentando una evolución positiva tras la crisis experimentada en el año 2008, cuando solamente representaban el 5,3\% del crédito total. Paralelamente al avance de la participación en el crédito, se observa una caída en el número de entidades de crédito cooperativo ( 83 a 62 en el mismo periodo 2008-2018). Esta reducción se ha debido básicamente a procesos de concentración, mucho menos intensos que los experimentados por el resto del sistema bancario, en coherencia con su identidad territorial. De hecho, de las entidades existentes en 2018, 40 de ellas tienen un ámbito local o comarcal, mientras que 22 presentan un carácter provincial o superior, entre las que se incluyen las cuatro cooperativas de crédito "no rurales" (profesionales y populares).

Por otra parte, se pone de manifiesto la diferencia existente en torno a los patrones de comportamiento de este tipo de entidades frente al resto, lo que permite la creación y consolidación del capital social resultante de las relaciones entre cooperativas y stakeholders (Marcuello y Saz, 2008), favoreciendo a su vez los procesos de innovación social (Vézina et al., 2017, Sanchis y Campos, 2008). En este sentido, atendiendo al comportamiento y orientación de negocio, distinguimos la banca de inversión, la mayorista, la diversificada y la centrada en el cliente minorista Ayadi (2017). De igual forma, Goglio y Alexopoulos (2012) distinguen entre dos tipos de instituciones, las que persiguen maximizar el shareholder value, es decir, la maximización de beneficios para los propietarios-accionistas y las que buscan la maximización del valor (stakeholder value) para todos los interesados.

Debemos hacer notar que incluso los sistemas financieros más desarrollados, no han sido completamente exitosos a la hora de alcanzar la inclusión financiera total de ciertos segmentos de población (Sarma, 2012). En este sentido, las entidades de crédito han de asumir la responsabilidad de la inclusión financiera, esto es, facilitar el acceso a unos servicios bancarios adecuados a las necesidades de la población, sobre todo en aquellos territorios en declive económico y demográfico. Se plantea, así, la hipótesis de que las entidades de crédito orientadas al stakeholder value y que cuentan con una mayor vocación regional, actúan impulsando la inclusión financiera, lo que permitiría frenar los procesos de despoblación.

La definición de inclusión financiera (y por oposición la de exclusión) varía según los autores o instituciones de referencia (Park y Mercado, 2018). Por lo general, se acepta que la inclusión financiera supone el acceso a servicios e instrumentos de transacciones, ahorro, crédito, seguros, pensiones, etc y que dicho acceso debe favorecer la mejora de la vida de los individuos (Zhu y Walker, 2018; Gitaharie, Soelistianingsih and Djutaharta, 2018; Demirgüç-Kunt et al. 2018) y su inclusión social, pues, al facilitar la inversión en salud, educación y negocios, puede evitar la pobreza de los individuos y ayuda a manejar emergencias financieras (como perder el empleo o la cosecha), favoreciendo por tanto la inclusión social (Demirgüç-Kunt, Klapper y Singer, 2017).

Centrándonos en el fenómeno de la exclusión financiera, nos podemos encontrar con distintas variantes en torno a éste (Kempson y Hhyley, 1999ª y1999b; Devlin, 2009). Así se puede hablar de exclusión de acceso, relacionada con la falta de disponibilidad de servicios financieros como consecuencia de la menor presencia de oficinas bancarias; exclusión de condición, relacionada con el precio de los productos financieros ofrecidos; exclusión de mercado o de marketing, producida cuando ciertos colectivos son ignorados por parte de las entidades bancarias, y por último puede llegar a existir autoexclusión fruto de la negativa impresión sobre el mercado bancario.

Por su parte, la exclusión financiera de tipo geográfica es más intensa donde la densidad de población es menor (Alamá y Tortosa-Ausina, 2012; Maudos, 2017), pasando a existir una relación directa entre el número de oficinas bancarias y la densidad de población. De esta forma, en aquellos territorios donde la densidad de población es baja cabe de esperar que también lo sean el número de oficinas bancarias. Como reflejo, el crédito por habitante en la "España vaciada" era en 2018 era el 59\% respecto a la media nacional. La extraordinaria diversidad en la densidad de población se traduce en una concentración de servicios bancarios en determinadas áreas, disminuyendo el atractivo para los proveedores de servicios financieros allí donde la población goza de menor densidad y renta (Daneshvar et al., 2017), limitando la satisfacción de necesidades de la población afectada. Así lo destaca Maudos (2017), indicando que el número de municipios que no cuentan con ninguna oficina aumentó en 124 entre los años 2008 y 2012, lo que supone un incremento de casi 100.000 personas que pasan a estar excluidas financieramente en este periodo de tiempo, situación que se ve agravada en el medio rural donde la dimensión del mercado potencial es cada vez menor fruto de las características sociodemográficas predominantes. 
En este contexto de creciente exclusión financiera, las cooperativas de crédito están presentes en las zonas rurales, aprovechando los nichos de mercado que dejan el resto de los bancos comerciales, dotando de servicios financieros a aquellos municipios carentes de oficinas bancarias. Así, autores como McKillop et al., 2007; McKillop et al., 2011 destacan el papel protagonista de este tipo de entidades en la lucha contra la exclusión financiera, la disponibilidad de crédito y la provisión de servicios. En este sentido, tal es el protagonismo de este tipo de entidades que a nivel municipal las cajas de ahorros rescataban a 993 municipios, las cooperativas de crédito a 301, mientras que los bancos comerciales solo lo hacían en 122 (Maudos, 2017), lo que pone de manifiesto una vez más el papel determinante que tienen las entidades de crédito orientadas a los stakeholders en el fenómeno de la inclusión financiera. De esta forma, las cooperativas de crédito favorecen el desarrollo de las regiones españolas en declive demográfico (Giagnocavo et al., 2012).

En España se evidencia el mayor peso de las cooperativas de crédito en aquellas regiones donde la densidad de población es menor (vid tabla 4.1), es decir, donde los procesos de despoblación generalmente son más intensos. Este hecho permite observar lo descrito con anterioridad, y es que, las cooperativas de crédito se instalan en los nichos de mercado que deja el resto del sistema bancario, prestando servicios financieros en aquellos espacios donde el riesgo de exclusión financiera es elevado. Así, presentan una presencia superior al $12 \%$ en provincias como Albacete, Almería, Álava, Asturias, Badajoz, Burgos, Castellón, Ciudad Real, Cuenca, Gipuzkoa, Granada, Huelva, Huesca, Jaén, Murcia, Navarra, Palencia, Rioja, Salamanca, Soria, Teruel, Toledo, Valladolid y Zamora, provincias que en su mayoría sufren despoblación.

Tabla. 4.1: Cuota de mercado de cooperativas por provincia respecto al crédito bancario total

\begin{tabular}{|lrr|lrr|}
\hline Provincias & Cuota de mercado & & Provincias & Cuota de mercado \\
Albacete & 2008 & 2018 & & 2008 & 2018 \\
Alicante & $12,24 \%$ & $33,84 \%$ & Jaén & $10,04 \%$ & $16,89 \%$ \\
Almería & $4,71 \%$ & $5,54 \%$ & León & $5,38 \%$ & $10,14 \%$ \\
Álava & $28,22 \%$ & $43,69 \%$ & Lleida & $2,18 \%$ & $5,33 \%$ \\
Asturias & $18,04 \%$ & $22,90 \%$ & Lugo & $4,22 \%$ & $9,36 \%$ \\
Ávila & $9,46 \%$ & $14,45 \%$ & Madrid & $0,62 \%$ & $1,28 \%$ \\
Badajoz & $4,22 \%$ & $11,65 \%$ & Málaga & $8,25 \%$ & $9,02 \%$ \\
Balears & $7,94 \%$ & $12,87 \%$ & Murcia & $11,97 \%$ & $18,17 \%$ \\
Barcelona & $1,24 \%$ & $2,15 \%$ & Navarra & $25,66 \%$ & $33,95 \%$ \\
Bizkaia & $1,09 \%$ & $1,60 \%$ & Ourense & $0,81 \%$ & $3,18 \%$ \\
Burgos & $11,61 \%$ & $11,94 \%$ & Palencia & $8,01 \%$ & $12,53 \%$ \\
Cáceres & $8,32 \%$ & $14,20 \%$ & Palmas, Las & $3,08 \%$ & $5,09 \%$ \\
Cádiz & $1,79 \%$ & $5,53 \%$ & Pontevedra & $0,08 \%$ & $0,43 \%$ \\
Cantabria & $4,42 \%$ & $5,62 \%$ & Rioja & $9,97 \%$ & $17,26 \%$ \\
Castellón & $2,42 \%$ & $3,12 \%$ & Salamanca & $9,47 \%$ & $14,21 \%$ \\
Ciudad Real & $14,01 \%$ & $19,46 \%$ & Tenerife & $4,59 \%$ & $9,95 \%$ \\
Córdoba & $10,40 \%$ & $19,89 \%$ & Segovia & $4,03 \%$ & $11,93 \%$ \\
Coruña & $5,06 \%$ & $8,51 \%$ & Sevilla & $3,48 \%$ & $5,37 \%$ \\
Cuenca & $0,10 \%$ & $0,71 \%$ & Soria & $29,35 \%$ & $48,28 \%$ \\
Gipuzkoa & $14,17 \%$ & $29,53 \%$ & Tarragona & $1,60 \%$ & $2,36 \%$ \\
Girona & $18,85 \%$ & $25,13 \%$ & Teruel & $25,39 \%$ & $39,64 \%$ \\
Granada & $0,31 \%$ & $0,70 \%$ & Toledo & $9,50 \%$ & $16,82 \%$ \\
Guadalajara & $14,98 \%$ & $21,44 \%$ & València & $8,88 \%$ & $11,16 \%$ \\
Huelva & $1,93 \%$ & $5,87 \%$ & Valladolid & $9,68 \%$ & $13,83 \%$ \\
Huesca & $9,43 \%$ & $13,19 \%$ & Zamora & $26,55 \%$ & $39,65 \%$ \\
ESPAÑA & $12,97 \%$ & $14,12 \%$ & Zaragoza & $12,23 \%$ & $11,38 \%$ \\
\hline & $5,33 \%$ & $7,43 \%$ & & & \\
\hline
\end{tabular}

Fuente: Elaboración propia a partir de datos de UNACC y Banco de España (2019) 
Este fenómeno se puede apreciar con claridad en el gráfico 4.1, a partir de la relación entre cuota de mercado de las cooperativas de crédito y la ratio empleo/superficie, indicador este último del grado de despoblación efectiva.

Gráfico. 4.1. Relación entre empleo/superficie y cuota de mercado de cooperativas (2018)

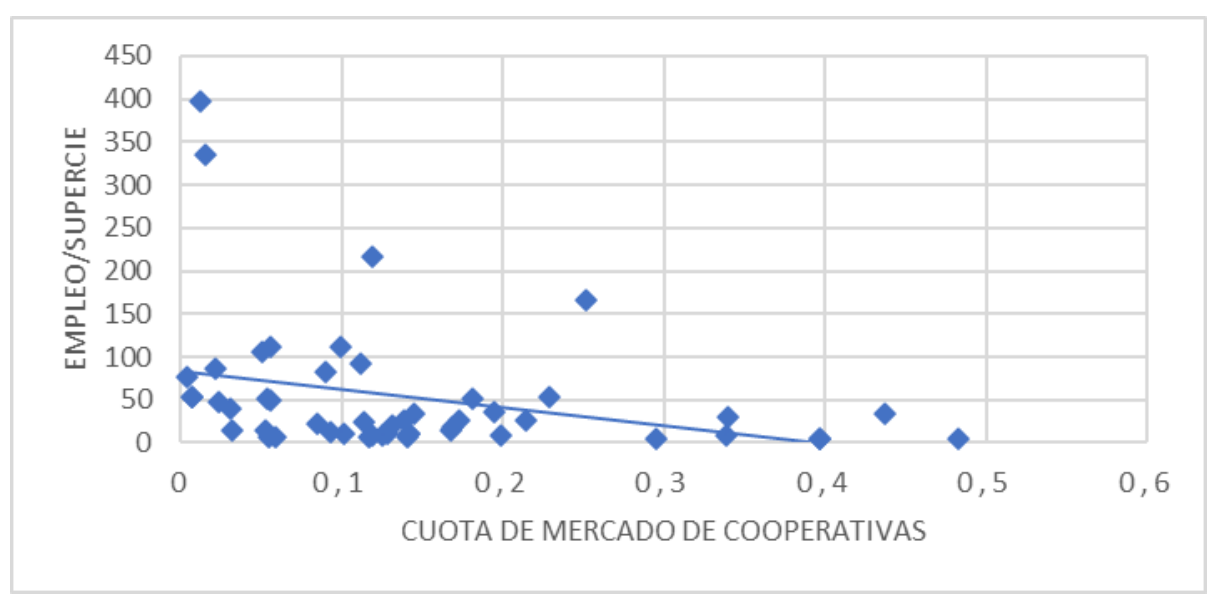

Fuente: Elaboración propia a partir de datos de INE, Banco de España, UNACC y ABC, 11/03/2019

En primer lugar, observamos que las cooperativas de crédito muestran una presencia más significativa en las provincias con mayores problemas de despoblación, medida a través de empleo por kilómetro cuadrado. En este sentido, existe una correlación claramente negativa entre cuota de mercado de las cooperativas de crédito y densidad de empleo, con la destacada excepción del País Vasco, donde tanto la presencia del cooperativismo como la ratio empleo/superficie son superiores a la media. Este hecho, permite suponer que la mayor cuota de mercado de las cooperativas de crédito en las provincias más deprimidas contribuye a evitar la exclusión financiera, favoreciendo la dinámica del desarrollo regional, ya que su carácter de banca de proximidad contribuye a canalizar el ahorro hacia su entorno más próximo.

Ahora bien, para valorar en qué medida las cooperativas de crédito contribuyen a mitigar o ralentizar el proceso de retroceso del crédito en los territorios en riesgo de marginalidad se puede utilizar un índice de intensidad de crédito per cápita, definido como el cociente entre la cuota de crédito de un territorio partido por la cuota de población de éste (García Verdugo y De la Cuesta, 1999).

$$
I I C P o b_{i}=\frac{\frac{\text { Crédito bancario provincia } i}{\text { Crédito bancario total }(\text { Españ } a)}}{\frac{\text { Población provincia } i}{\text { Población total }(\text { España })}}=\frac{\frac{C B_{i}}{C B T}}{\frac{P_{i}}{P T}}
$$

El índice de intensidad de crédito (cuota crédito/cuota población) para el global del Estado es, por definición 1. Pero para el conjunto de la España vaciada es tan solo del $60 \%$ y ninguna de las veintiuna provincias consideradas en esta categoría tiene un índice superior a 1 en 2018 (Tabla 4.2).

En general, el sistema bancario tiende a exacerbar las diferencias regionales, al concentrar progresivamente el crédito en aquellos territorios más pujantes económicamente, de modo que los territorios con menores tasas de empleo por kilómetro cuadrado serán menos atractivos para el crédito bancario, favoreciendo la dispersión de este índice de intensidad de crédito, acentuando el círculo vicioso que aleja la financiación de las zonas menos favorecidas y limitando las posibilidades de desarrollo económico de las mismas. Por su parte, las cooperativas de crédito han venido actuando como un contrapeso a esa tendencia. De hecho, observando el gráfico 4.2, que nos muestra la relación existente entre la variación de intensidad de crédito y la variación con respecto a la cuota de mercado de las cooperativas para el periodo 2008-2018, se aprecia una relación inversa entre ambas variables, es decir, las provincias que han visto disminuida la intensidad de crédito en menor medida que el resto se corresponden con aquellas donde la presencia de crédito cooperativo es creciente, lo que nos permite aceptar la hipótesis de que este tipo de entidades actúan impulsando la inclusión financiera en los territorios más vulnerables, lo que a su vez permite frenar los procesos de despoblación.

De hecho, la década 2008-2018 estuvo marcada por un duro ajuste de la crisis económica, que se tradujo en un retroceso del crédito concedido por el sistema bancario del 3,9\% anual, generalizado en todas las provincias españolas. La caída del crédito bancario fue aún más acusada en las provincias de la "España 
vaciada", alcanzando un 5,0\% anual (frente a un descenso medio estatal del 3,9\%), lo que refleja la tendencia indicada de declive económico.

No obstante, si se analizan los mismos indicadores referidos exclusivamente a la financiación concedida por cooperativas de crédito se aprecia algunas diferencias significativas. En primer lugar, la caída del crédito fue significativamente inferior, tanto en el conjunto estatal $(0,6 \%)$, como para la "España vaciada" $(1,5 \%)$. En segundo lugar, este retroceso de la actividad crediticia no fue generalizado, concentrándose en determinadas provincias que sufrieron la debacle inmobiliaria (disminución superior al 3\% anual en Castellón, Zaragoza, Málaga, València y Huesca). Por el contrario, teniendo en cuenta que el crédito bancario total cayó en la totalidad de las provincias españolas, en 15 de las 21 provincias de la "España vaciada" el crédito concedido por cooperativas, lejos de disminuir aumentó, destacando los casos de Albacete, Segovia y las provincias gallegas. En tercer lugar, entre las 11 provincias de la "España vaciada" con menor intensidad de crédito (inferior al 60\%), las cooperativas aumentaron el volumen de préstamos de forma significativa en la mayor parte de ellas, salvo en León y Palencia, en las que hubo estabilidad, y Zamora, con un ligero retroceso.

De esta forma, el crédito cooperativo ha contribuido a frenar el deterioro de las regiones más desfavorecidas, a consolidar su estructura productiva y a vertebrar un tejido empresarial que a su vez permita afianzar vínculos propios del capital social. Pero no solo eso, además han venido a compensar el progresivo abandono de estos territorios por parte del sistema bancario lucrativo, sobre todo con la desaparición y transformación de las cajas de ahorros y la concentración en unos pocos grupos bancarios. Todo ello sin pretender desdeñar el hecho de que también el propio sector de las cooperativas de crédito ha sufrido el ajuste que ha propiciado la desaparición de algunas cajas de carácter local, absorbidas por otras de mayor tamaño (Palomo y Sanchis, 2010).

Gráfico. 4.2. Relación entre variación de intensidad de crédito y variación cuota de mercado de cooperativas (20082018)

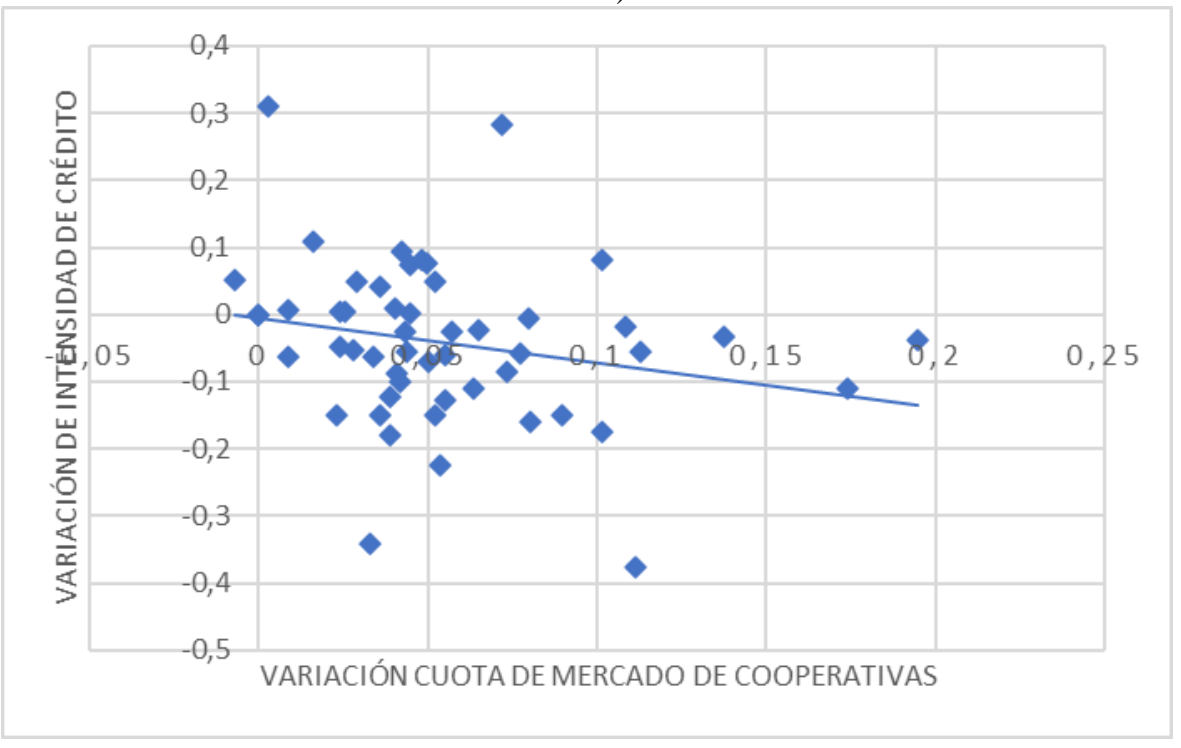

Fuente: Elaboración propia a partir de datos INE, Banco de España y UNACC

En consecuencia, se observan las diferencias existentes con respecto a los patrones de comportamiento financiero, como predecían Goglio y Alexopoulos (2012). Mientras que las principales entidades de crédito, cuyo objetivo es la maximización de beneficios para los accionistas, se instalan en aquellas provincias que gozan de una amplia y consolidada estructura demográfica y económica, las cooperativas de crédito, persiguiendo su objeto social de servir a las necesidades financieras de sus socios y de terceros, ocupan los nichos de mercado abandonados por el resto de entidades, instalándose en aquellas provincias donde los procesos de pérdida de población son más intensos. Del mismo modo, observamos cómo las cooperativas de crédito contribuyen en la lucha de la exclusión financiera, sobre todo de tipo geográfica, al seguir un comportamiento contrario al de la banca mayorista o de inversión. Este hecho es observable a través de la mayor cuota de mercado existente en aquellas provincias que cuentan con una densidad de población menor y la evolución del crédito en los años de depresión, dando lugar a una mayor diversidad y disponibilidad de servicios financieros en aquellas zonas en riesgo de exclusión. Así, la propia razón de ser y características de las entidades de crédito cooperativo rompen la tendencia a relegar las zonas de menor densidad demográfica 
ante el escaso el atractivo para los proveedores de servicios financieros (Daneshvar et al., 2017; Maudos, 2017; McKillop et al., 2007; McKillop et al., 2011; Ureña et al., 2013).

Estos hechos mitigan los círculos viciosos de crecimiento mencionados con anterioridad, contribuyendo al fortalecimiento de las relaciones de confianza dentro del territorio, dados los valores de proximidad y compromiso con el entorno y su clientela que caracterizan a estas instituciones de Economía Social. En este sentido, actúan como agentes dinamizadores de recursos y activos locales al facilitar la financiación del desarrollo económico, contribuyendo a la generación de nuevos flujos de actividad económica, a la dinamización económica de las regiones, a la generación de vínculos empresariales que generan cohesión interna territorial y a un mayor bienestar social, favoreciendo en última instancia la convergencia regional.

Tabla. 4.2: Índice de intensidad de crédito por provincias.

Datos superiores a la unidad indican que el crédito bancario por persona es superior a la media estatal. Sombreadas las provincias seleccionadas con empleo inferior a 20 empleos $/ \mathrm{km}^{2}$

\begin{tabular}{|lrr|lrr|}
\hline Provincias & Índice & & & Provincias & Índice \\
Albacete & 2008 & 2018 & & 2008 & 2018 \\
Alicante & 0,64 & 0,73 & Jaén & 0,48 & 0,53 \\
Almería & 0,77 & 0,88 & León & 0,67 & 0,56 \\
Álava & 0,99 & 0,93 & Lleida & 0,88 & 0,73 \\
Asturias & 1,05 & 1,06 & Lugo & 0,41 & 0,40 \\
Ávila & 0,64 & 0,73 & Madrid & 1,83 & 2,11 \\
Badajoz & 0,71 & 0,54 & Málaga & 0,78 & 0,72 \\
Balears & 0,54 & 0,62 & Murcia & 0,90 & 0,80 \\
Barcelona & 1,05 & 0,99 & Navarra & 1,01 & 0,96 \\
Bizkaia & 1,29 & 1,17 & Ourense & 0,42 & 0,39 \\
Burgos & 1,06 & 1,37 & Palencia & 0,57 & 0,57 \\
Cáceres & 1,01 & 0,78 & Palmas, Las & 0,75 & 0,68 \\
Cádiz & 0,49 & 0,44 & Pontevedra & 0,71 & 0,60 \\
Cantabria & 0,59 & 0,54 & Rioja & 1,04 & 0,91 \\
Castellón & 0,71 & 0,71 & Salamanca & 0,73 & 0,65 \\
Ciudad Real & 1,13 & 0,79 & Tenerife & 0,66 & 0,61 \\
Córdoba & 0,57 & 0,54 & Segovia & 0,67 & 0,65 \\
Coruña & 0,74 & 0,59 & Sevilla & 0,81 & 0,79 \\
Cuenca & 0,71 & 0,67 & Soria & 0,64 & 0,71 \\
Gipuzkoa & 0,68 & 0,59 & Tarragona & 0,89 & 0,71 \\
Girona & 0,94 & 0,99 & Teruel & 0,51 & 0,58 \\
Granada & 0,89 & 0,73 & Toledo & 0,70 & 0,67 \\
Guadalajara & 0,76 & 0,61 & València & 1,02 & 0,86 \\
Huelva & 1,06 & 0,69 & Valladolid & 0,87 & 0,91 \\
Huesca & 0,61 & 0,54 & Zamora & 0,49 & 0,50 \\
ESPAÑA & 0,75 & 0,76 & Zaragoza & 1,04 & 1,09 \\
\hline
\end{tabular}

Fuente: Elaboración propia a partir de datos de UNACC y Banco de España (2019)

\section{Conclusiones}

La pasada recesión económica que han padecido la mayoría de los países desarrollados en general, ha puesto de relieve las diferencias existentes entre la banca mercantil-capitalista y la banca cooperativa a la hora de hacer frente a los efectos nocivos de la misma, pues los propios valores cooperativos, junto con la clara vocación territorial y de proximidad con la que cuentan este tipo de instituciones, se han convertido en los pilares fundamentales para permanecer al margen de las turbulencias derivadas de la crisis. Esta dualidad 
de objetivos se traduce en una mayor relación con el entorno inmediato, una menor propensión al riesgo y, en definitiva, una mayor estabilidad, a pesar de sus evidentes limitaciones.

Las dificultades de los grandes bancos han generado oportunidades para pequeñas entidades de banca de proximidad, que han cubierto los espacios abandonados ante su menor rentabilidad. Por otra parte, la diferente orientación de negocio y sus singulares características jurídicas y operativas refuerzan el papel de estas instituciones a la hora de otorgar una mayor diversidad y, por tanto, resiliencia, al conjunto del sistema financiero. Sin embargo, las cooperativas de crédito presentan una pequeña participación en la actividad financiera (en torno al 6\% del total de sistema bancario español).

Aun teniendo en cuenta estos datos, el presente artículo revela la importancia que tienen este tipo de entidades cooperativas en la lucha contra la exclusión financiera, sobre todo en las provincias de la "España Vaciada", donde actúan financiando el desarrollo económico de la zona mediante la movilización de los recursos y activos locales, contribuyendo al equilibrio regional. En esta misma línea, las cooperativas de crédito canalizan el ahorro hacia la propia región, asegurando la reinversión de sus fondos dentro de ésta. Además, favorecen la creación de redes de empresas, la consolidación de capital social, así como la formación y consolidación de nuevas estructuras productivas, que son piezas claves para la dinamización económica del entorno.

Así, en aquellas regiones donde la densidad de población es menor, y los problemas de despoblación son más acusados, el estudio nos ha permitido demostrar que la existencia de entidades de la Economía Social, como son las cooperativas de crédito, supone un factor de movilización de los recursos productivos locales, lo que favorece la actividad económica en diferentes sectores de actividad y la generación de empleo estable y de calidad, que contribuye a fijar la población en estos territorios.

Finalmente, el escenario incierto que se avecina tras el impacto de la crisis sanitaria del Covid añade un elemento crucial de preocupación para el futuro de estas entidades. Por una parte, se ha producido una mayor atención hacia la olvidada España rural y se ha revalorizado el papel del sector primario, como elemento estratégico. Por otra parte, los efectos sobre las entidades de crédito en general no apuntan al optimismo: el previsible aumento de la morosidad sin haber superado los efectos de la anterior crisis financiera, la caída brusca de la rentabilidad, los tipos de interés persistentemente bajos y la atonía económica, son elementos que obligan a un cuestionamiento del modus operandi de la banca. La respuesta de mejorar la eficiencia mediante la concentración puede generar un riesgo de oligopolización y de reducción de diversidad, que a la postre pueden debilitar la estabilidad del conjunto del sistema. El cooperativismo de crédito se encuentra en la encrucijada de un dilema estratégico: seguir la estela del sistema bancario de concentrarse para competir con mayor eficiencia o perseverar en un modelo territorial y de proximidad. Cabe también la alternativa de profundizar en la intercooperación, y en la consolidación como grupo, manteniendo su vocación de garantizar la integración financiera y el bienestar de la gente de su territorio.

Se abren, por tanto, interesantes temas de investigación futura en torno a la cooperación de crédito, todos ellos relacionados con la resiliencia de los espacios rurales, su inclusión financiera y el sostenimiento de la población rural, como son las alianzas entre cajas rurales y cooperativas agrarias; el proceso de concentración de cooperativas de crédito, provocado por la crisis de 2008 y su impacto en la actividad financiera de las cajas rurales y su ámbito de actuación; el papel de las redes sociales y la innovación social; la utilización del Fondo de Educación, Formación y Promoción Cooperativa, etc.

\section{Referencias bibliográficas}

Alamá, L. y Tortosa-Ausina, E. (2012) Bank Branch Geographic Location Patterns in Spain: Some Implications for Financial Exclusion, Growth and Change, vol. 43, № 3, pp. 505-543, DOI: 10.1111/j.1468-2257.2012.00596.x.

Almond, G. A. y Verba, S. (1963) The civic culture: Political attitudes and democracy in five nations. London: Sage Publications

Ammirato, P. (2018) The growth of italian cooperatives: innovation, resilience and social responsibility. Oxon, Abingdon: Routledge.

Arnone, M. (2015) Il credito cooperative negli anni della crisi: un'analisi territoriale, Economia Cooperativa, pp. 192200.

Ayadi, R. (2017) Banks' business models in Europe: Are cooperative banks different? En E. Miklaszewska(ed.). Institutional diversity in banking: small country, small banks perspectives. London: Palgrave Macmillan.

Ayuda, M. I., Navarro, V., y Sáez, L. A. (2000) El problema de la despoblación en Aragón: causas, características y perspectivas. Revista de Demografía Histórica, vol. 18, № 1, pp. 137-173.

Azmi, A. (2015) Cooperative Finance and Sustainability After the Financial Crisis. En Finance and Sustainability: Towards a New Paradigm? A Post-Crisis Agenda (Critical Stuidies on Coprporate Responsibility, Governance and Sustainability). Bingley: Emerald Group Publishing Limited, DOI: 10.1108/S2043-9059(2011)0000002017. 
Bauer, C.M., Guzmán, C. y Santos, F. J. (2012) Social capital as a distinctive feature of Social Economy firms. International Entrepreneurship and Management Journal, $\mathrm{N}^{\circ}$ 8, pp. 437-448.

Bayona, J., y Gil Alonso, F. (2010) Migraciones de españoles y extranjeros en las áreas rurales catalanas (1996-2006) ¿Fin de la despoblación o nuevo modelo territorial?. Boletín de la Asociación de Geógrafos Españoles, № 53, pp. 219-237.

Belmonte Ureña, L.J. (2007) El sector de cooperativas de crédito en España: un estudio por comunidades autónomas. Sevilla: Consejo Económico y Social de Andalucía.

Bourdieu, P. (1980) Le capital social. Notes provisoires. Actes de la Recherche en Sciences Sociales, No 31, pp. 2-3.

Bretos, I. y Errasti, A. (2017) Challenges and Opportunities for the Regeneration of Multinational Worker Cooperatives: Lessons from the Mondragon Corporation-A Case Study of the Fagor Ederlan Group. Organization, vol. 2, N²4, pp. 154-173.

Bretos, I., Díaz-Foncea, M. Marcuello, Ch. y Marcuello, C. (2018) Cooperativas, capital social y emprendimiento: Una perspectiva teórica. REVESCO. Revista de Estudios Cooperativos, Segundo Cuatrimestre, No 128, pp. 76-98. DOI: http://dx.doi.org/10.5209/REVE.59775.

Calderón, B. y Calderón, M. J. (2012) La calidad del empleo de las entidades de la economía social en período de crisis. EKONOMIAZ. Revista vasca de Economía, vol. 79, № 1, pp. 31-58.

Camarero, L. y Sampedro, R. (2019) Despoblación y ruralidad transnacional: crisis y arraigo rural en Castilla y León. Economía Agraria y Recursos Naturales, vol. 19, № 1, pp. 59-82.

Carchano, M y Carrasco, I. (2020) La Economía Social y la industria agroalimentaria como factores de resiliencia de la población en Castilla-La Mancha, en G. Fajardo y J. Escribano (coords.) Despoblamiento y Desarrollo Rural. Propuestas desde la Economía Social. Valencia: IUDESCOOP y CIRIEC-España (En prensa).

Carrasco, I. (2005) Ética, cultura y economía. ICE, Revista De Economía, No 823, pp.189-198.

Carrasco, I. y Buendía-Martínez, I. (2013) El tamaño del sector cooperativo en la Unión Europea: una explicación desde la teoría del crecimiento económico. CIRIEC-España. Revista de Economía Pública, Social y Cooperativa, $\mathrm{N}^{\mathrm{o}} 78$, pp. 125-148.

Castaño, M. S. (2005) Los valores éticos del capital social y su influencia en el crecimiento económico. ICE, Revista De Economía, No 823 , pp. 131-140.

Chaves, R. \& Monzón, J.L. (2007) La Economía Social en la Unión Europea. Recuperado de: www.eesc.europa.eu/groups/3/index en.asp?id=1405GR03EN.

Chaves, R. y Soler, F. (2005) El comportamiento de cajas de ahorros y cooperativas de crédito españolas frente a los fines sociales: la obra benéfico-social y el fondo de educación y promoción cooperativa. GEZKI, Revista Vasca de Economía Social, № 1, pp. 45-62.

CES. Consejo Económico y Social de España (2018) Informe CES 1/2018. El medio rural y se vertebración social y territorial. Colección Informes, 01/2018.

Coleman, J. S. (1988) Social Capital in the creation of Human Capital, American Journal of Sociology, vol 94.

Coleman, J. S. (1990) The Foundations of Social Theory. Cambridge: Harvard University Press.

Daneshvar, C., Garry, S., López, J., Santamaría, J. y Villareal, F.G. (2017) Financial inclusion of small-scale rural producers: trends and challenges. En F.G.Villareal (ed.) Financial inclusion of small rural producers. Santiago: United Nations, ECLAC.

Demirgüç-Kunt, A.; Klapper, L. y Singer, D. (2017) Financial Inclusion and Inclusive Growth: A Review of Recent Empirical Evidence, Policy Research Working Paper 8040, World Bank, Washington, DC

Demirgüç-Kunt, A.; Klapper, L.; Singer, D.; Ansar, S. y Hess, J.(2018) The Global Findex Database 2017: Measuring Financial Inclusion and the Fintech Revolution. Washington, DC: World Bank.

Devesa, J. E. (2011) Hacia una economía con rostro humano: el capital social en acción para superar la crisis. Boletín Red Iberoamericana de Universidades por la Responsabilidad Social Empresarial, vol. 2, pp. 1-17.

Devlin, J.F. (2009) An Analysis of Influences on Total Financial Exclusion, Service Industries Journal, No 29, pp 10211036. DOI: $10.1080 / 02642060902764160$.

Eversole, R., Barraket, J. y Luke, B. (2013) Social enterprises in rural community development. Community Development Journal, vol. 49, No 2, pp. 245-261.

Fernández, M. y Meixide, A. (2010) Migraciones de españoles y extranjeros en las áreas rurales catalanas (1996-2006) ¿Fin de la despoblación o nuevo modelo territorial?. Boletín de la Asociación de Geógrafos Españoles, № 53, pp. 219-237.

Ferragina, E. (2012) Social capital in Europe: A comparative regional analysis. Cheltenham: Edward Elgar Publishing.

Foronda-Robles, C., y Galindo-Pérez de Azpillaga, L. (2012) Argumentación relativa a la confianza territorial. Claves sobre capital social. Cuadernos de desarrollo rural, vol. 9, № 68, pp. 41-63.

Fukuyama (1995) Social capital and the global economy. Foreign Affaire, vol. 74, № 5, pp. 89-103.

García Verdugo, J. y De la Cuesta, M. (1999) Efectos del comportamiento diferencial de las entidades bancarias sobre la distribución espacial del crédito. Cuadernos de Información Económica, № 145, pp. 138-149.

García, R. y Espejo, C. (2019) Los círculos viciosos de la despoblación en el medio rural español: Teruel como caso de estudio. Revista de Estudios Geográficos, vol. 80, № 286, pp. 9-13.

Garrido, J. y Faci, Y. (2004) Causas de la despoblación en la cuenca del río Guadalope: comarcas del bajo Aragón y Maestrazgo propuesta de políticas demográficas y de desarrollo endógeno. Informes del CEDDAR, $\mathrm{N}^{\circ} 1$, pp. 1-134. 
Giagnocavo, C., Gerez, S. y Sforzi, J. (2012) Cooperative bank strategies for social-economic problem solving: supporting social Enterprise and local development, Annals of Public and Cooperative Economics, vol. 83, № 3, pp. 281-315.

Gómez-Limón, J. A., Atance, I. y Rico, M. (2007) Percepción pública del problema de la despoblación del medio rural en Castilla y León. Ager: Revista de estudios sobre despoblación y desarrollo rural= Journal of depopulation and rural development studies, $\mathrm{N}^{\circ}$ 6, pp. 9-60.

Gitaharie, T., Soelistianingsih, L., y Djutaharta, T. (2018) Financial inclusion: The impacts of bank loans on household welfare, en Gani et al. (Eds) Competition and Cooperation in Economics and Business, Londres: Taylor \& Francis Group, ISBN 978-1-138-62666-9.

Grootaert, C., Narayan, D., Jones, V. N., y Woolcock, M. (2004) Measuring social capital: An integrated questionnaire. The World Bank.

Haub, C. y Toshiko K. (2014) World Population Data Sheet. Washington, DC: Population Reference Bureau.

Hauberer, J. (2011) Social Capital Theory. Towards a Methodological Foundation. Wiesbaden: VS Verlag für Sozialwissenschaften.

Hogeland, J.A. (2006) The economic culture of U.S. agricultural cooperatives. Culture \& Agriculture, No 28, pp. 67-79.

Inkpen, A. y Tsang, F. (2005) Social capital, networks, and knowledge transfer. Academy of Management Review, vol. 30, No 1, pp. 146-165. DOI: 10.2307/20159100.

Johnson, K., Layton, F. y Dudley, P. (2015) More Deaths than Births: Subnational Natural Decrease in Europe and the United States. Population and Development Review, vol. 41, № 4, pp 651-80.

Kasimimis, C. (2010) Demographic Trends in rural Europe and international migration to rural areas. Agriregionieuropa, vol. 21, № 6, pp. 1-6.

Kempson, E. y Whyley, C. (1999a) Kept in or Opted Out? Understanding and Combating Financial Exclusion. Bristol: Policy Press.

Kempson, E. y Whyley, C. (1999b) Understanding and Combating Financial Exclusion, Insurance Trends, $\mathrm{N}^{\mathrm{o}} 21, \mathrm{pp}$. 18-22.

Marcuello, C. y Saz, M. I. (2008) Los principios cooperativos facilitadores de la innovación: Un modelo teórico, REVESCO. Revista de Estudios Cooperativos, Primer Cuatrimestre, № 94, pp. 59-79.

Margaras, V. (2019) Demographic trends in EU regions", European Parliamentary Research Service. Recuperado de: https://ec.europa.eu/futurium/en/system/files/ged/eprs-briefing-633160-demographic-trends-eu-regions-final.pdf.

Marré, A. W. y Rupasingha, A. (2019) School quality and rural in-migration: Can better rural schools attract new residents?. Journal of Regional Science, pp 1-19. DOI: 10.1111/jors.12437.

Maudos, J. (2016) La concentración regional del mercado bancario, Cuadernos de Información Económica, Nº 251, pp. 49-62.

Mckillop, D.G. y Ward, A. (2011) An examination of volunteer motivation in credit unions: informing volunteer resource management, Annals of Public and Cooperative Economics, vol. 82, $\mathrm{N}^{\mathrm{o}}$ 3, pp. 253-275, DOI: 10.1111/J.1467-8292.2011.00438.X.

Mckillop, D.G., Ward, A. y Wilson, J. (2007) The Development of Credit Unions and Their Role in Tackling Financial Exclusion, Public Money \& Management, vol. 27, $\mathrm{N}^{\mathrm{o}}$ 1, pp. 37-44.

Melián-Navarro, A., Campos-Climent, V., \& Sanchis-Palacio, J. R. (2011) Análisis de las fusiones entre cajas rurales y su influencia en las cooperativas agrarias. El caso valenciano en España. INNOVAR. Revista de Ciencias Administrativas y Sociales, vol. 21, No 41, pp. 91-109.

Miner, R. (2018) Inmigrantes y refugiados en una España con fuerte despoblación rural y baja natalidad. En La acción social de la Iglesia: XIX Congreso Católicos y Vida Pública, Madrid: CEU Ediciones, D.L. 2018.

Mozas, A. y Bernal, E. (2006) Economía social y desarrollo rural. CIRIEC-España, Revista de Economía Pública, Social y Cooperativa, $\mathrm{N}^{\circ}$ 55, pp. 125-140.

Palomo Zurdo, R. J., Sanchis Palacio, J.R. y Soler Tormo, F. (2010) Las entidades financieras de economía social ante la crisis financiera: un análisis de las cajas rurales españolas. REVESCO. Revista de Estudios Cooperativos, $\mathrm{N}^{\circ} 100$, pp. 101-133.

Palomo Zurdo, R.J. y Sanchis Palacio, J.R. (2008) Un análisis del crédito cooperativo en España: Situación actual, expansión territorial y proyecciones estratégicas, Estudios de Economía Aplicada, vol. 26, Nº 1, pp. 89-132.

Palomo Zurdo, R. J. \& Sanchis Palacio, J. R. (2010) Efectos de las fusiones sobre la concentración y la eficiencia bancaria: el caso de las Cajas Rurales y los retos de la crisis financiera. Spanish Journal of Finance and Accounting/Revista Española de Financiación y Contabilidad, vol. 39, No 146, pp. 289-319.

Park, CY, y Mercado Jr, R. (2018) Financial Inclusion, Poverty, and Income Inequality. The Singapore Economic Review, Vol. 63, No 1, pp. 185-206.

Pérez, A. y Leco, F. (2011) Envejecimiento, estancamiento poblacional y perspectivas demográficas en Extremadura. Geographicalia, № 59 , pp. 309-321.

Pérez, A. (2006) Los pequeños municipios ante los retos del desarrollo. Norba. Revista de geografía, vol. 6, pp. 183197.

Pinilla, V. y Sáez, L.A. (2017) La despoblación rural en España: génesis de un problema y políticas innovadoras. Informes CEDDAR 2017-2. Zaragoza: Centro de Estudios sobre Despoblación y Desarrollo de Áreas Rurales.

Pisani, E., Franceschetti, G., Secco, L., y Christoforou, A. (2017) Social capital and local development. Basingstoke: Palgrave Editions. 
Putnam, R.D. (1993) The prosperous community: Social capital and public life. The American Prospect, № 13, pp. 3542.

Putnam, R.D. (2000) Bowling alone: the collapse and revival of American community. Simon and Schuster, New York.

Rajovig, G. y Bulatovic, J. (2013) Charateristics of Housing in Rural Villages: The Case Northeastern Montenegro. International Letters of Social and Humanistic Sciences, $\mathrm{N}^{\mathrm{o}}$ 6, pp. 24-35.

Rodríguez, Ó. (2006) El capital social como componente del capital intelectual de las cajas de ahorros, XVI Congreso Nacional de ACEDE, Universitat de València.

Sabatini, F. (2009) Social Capital as Social Networks: a New Framework for Measurement and an empirical analysis of its determinants and consequence. Journal of Socio-Economics, vol. 38, № 3, pp. 429-442.

Sabatini, F., Modena, F. y Tortia, E. (2014) Do cooperative enterprises create social trust?. Small Business Economics, $\mathrm{N}^{\mathrm{o}} 42$, pp. 621-64.

Sanchís Palacio, J. R. \& Campos Climent, V. (2007) La dirección estratégica en la economía social: utilización de herramientas de análisis estratégico en las cooperativas. CIRIEC-España, Revista de Economía Pública, Social y Cooperativa, $\mathrm{N}^{\circ}$ 59, pp. 237-258.

Sanchis Palacio, J.R. \& Campos Climent, V. (2008) La innovación social en la empresa: el caso de las cooperativas y de las empresas de economía social en España. Economía Industrial, Nº 368, pp. 187-196.

Sanchis Palacio, J. R., \& Melián Navarro, A. (2009) Rentabilidad y eficiencia de las entidades financieras de economía social en España. Revista Venezolana de Gerencia, vol. 14, No 45, pp. 24-41.

Sanchis Palacio, J.R. \& Melián Navarro, A. (2011) Strategic Diagnosis of Spanish Farming Cooperative Credit Sections: a SWOT Analysis. Annals of Public and Cooperative Economics, vol. 82, No 2, pp. 167-186.

Sarma, M. (2012) Index of Financial Inclusion - A measure of financial sector inclusiveness, Working Paper No. 07/2012. Berlin Working Papers on Money, Finance, Trade and Development.

Saz-Gil, M. I. y Gómez-Quintero, J.D. (2015) Una aproximación a la cuantificación y caracterización del capital social: una variable relevante en el desarrollo de la provincia de Teruel, España. EURE, vol. 41, No 123, pp. 29-51. https://dx.doi.org/10.4067/S0250-71612015000300002.

Seguí-Mas, E. y Server Izquierdo, R.J. (2010) El capital relacional de las cooperativas de crédito en España: un estudio cualitativo de sus intangibles sociales mediante el análisis Delphi. REVESCO. Revista de Estudios Cooperativos, Primer Cuatrimestre, № 101, pp. 107-131.

Server Izquierdo, R.J. y Melián, A. (1998) ooperativismo de crédito y secciones de crédito: Estrategias empresariales y caracterización socioeconómica. Revista española de estudios agrosociales y pesqueros, No 188, pp. 187-204.

Solow R. M. (2000) Notes on Social Capital and Economic Performance. En P. Dasgupta and I. Serageldin (eds.): Social Capital: A Multifaceted Perspective. Washington, D.C: World Bank

Surchev, P. (2010) Rural areas, problems and opportunities for development. Trakia Journal of Sciences, vol. 8, N 3 , pp. 234-239.

Sweeney, G. (2001) Social capital: the core factor in economic resurgence. En G. Sweeney (Ed.), Innovation, economic progress and the quality of life (pp. 144-163). Cheltenham: Edward Elgar.

Ureña, L.J., Álvarez, J.A. y Álvarez, J.F. (2013) La estrategia de concentración de las cooperativas de crédito españolas. Análisis de los sistemas institucionales de protección. Finanzas y Política Económica, vol. 5, № 2, pp. 23-36.

Valentinov, V. (2004) Toward a social capital theory of cooperative organization. Journal of Cooperative Studies, vol. $3, \mathrm{~N}^{\mathrm{o}} 37$, pp. 5-20.

Vézina, M., Malo, M.C. y Ben Selma, M. (2017) Mature social economy enterprise and social innovation: The case of Desjardins Environmental Fund, Annals of Public and Cooperative Economics, vol. 88, № 2, pp. 257- 278.

Whiteley, P.F. (2000) Economic growth and social capital. Political Studies, No 48, pp. 443-466.

Wu, W. P. (2008) Dimensions of social capital and firm competitiveness improvement: The mediating role of information sharing. Journal of management studies, vol. 45, $\mathrm{N}^{\mathrm{o}} 1$, pp. 122-146.

Zhu, H. y Walker, A.C. (2018) Pensions and social inclusion in an ageing China. Ageing \& Society. https://doi.org/10.1017/S0144686X17001593. Descargado el 09/10/2020. 